\title{
ONTOLOGICAL MODEL FOR CHARACTER RECOGNITION BASED ON SPATIAL RELATIONS
}

\author{
Aicha Eutamene, Mohamed Khireddine Kholladi and Hacene Belhadef \\ MISC Laboratory, NTIC Faculty, University of Constantine 2, Algeria \\ \{aicha.eutamene, kholladi, hacene_belhadef\}@umc.edu.dz \\ \{aicha.eutamene, kholladi, hacene_belhadef\}@yahoo.fr
}

\begin{abstract}
In this paper, we present a set of spatial relations between concepts describing an ontological model for a new process of character recognition. Our main idea is based on the construction of the domain ontology modelling the Latin script. This ontology is composed by a set of concepts and a set of relations. The concepts represent the graphemes extracted by segmenting the manipulated document and the relations are of two types, is-a relations and spatial relations. In this paper we are interested by description of second type of relations and their implementation by java code.
\end{abstract}

\section{KEYWORDS}

Ontology, Grapheme, Character Recognition, Semantic Gap \& Spatial Relations

\section{INTRODUCTION}

Handwritten character recognition is still a challenging problem for many languages like Farsi, Chinese, English, etc. Developing robust optical character recognition techniques would be very rewarding in today technology. Huge amount of research in this area has also contributed to solve other open problems in pattern recognition [1], such as the character recognition in old documents and word spotting technology.

The general goal of document recognition, whether printed or handwritten, is to turn it into a representation understandable and exploitable by machine. The process of recognition is not always easy as long as the content of the documents can have multiple representations.

Character recognition remains one of the vital research areas mainly over the past three decades. In recent years, ontologies have proven to be an important and useful tool for the representation, sharing and reuse of knowledge. Obviously also through ontology languages that can express a rich semantic and provide reasoning capabilities.

Knowledge representation under ontological form provides the following advantages: (1) it offers the possibility to establish a common understanding of the field of knowledge considered, (2) it allows representing knowledge in a form convenient for automated processing information and analysis systems, and provides an opportunity for the acquisition and accumulation of new knowledge and multiple use of knowledge. The association between graphic data and ontologies then allows to software- agents dash profit of the knowledge represented in ontologies to better exploit the images. So whatever the wealth of content from documents, this last remains insufficient to help the process to accomplish its task, so, an annotation step of the document is required to add additional information leading to the proper functioning of this process. The annotation of an image through the construction of ontologies is the main tool for associating

DOI : 10.5121/sipij.2013.4310 
semantics to an image and allows the use of research methods more powerful and capable of responding to complex queries.

The current revolution in the World Wide Web by using the notion of ontology has influenced in many domains of scientific research such that the domains that try bring a semantic to data and treatment; character recognition is one of these domains. In this paper, we tried to devise a new approach to character recognition (handwritten or printed) assisted by a domain ontology. The main idea is to model the domain of character recognition by a domain ontology, whose concepts are graphemes (defined hereinafter) extracted from a segmentation step and extraction primitive, these graphemes are interconnected by their spatial relationships showing their inter-location in the document and having both intrinsic and extrinsic properties describing their forms.

The use of ontology in such a process is justified by all the benefits of the latter, in order to improve the quality of results by introducing the concept of semantic and inference on facts that already exists. Our primary objective by given this proposition is to reduce and bridge the semantic gap between low-level knowledge provided by the image in the form of pixel and the high-level knowledge extracted and enriching the image. In the domain that we studied: the lower-level consists of several segments or primitives, characterized by low-level descriptors. The top level includes a generic model of knowledge "ontology" and an instantiated model of knowledge, valid for the vocabulary of the language written in the image being processed. So our approach is based on an ontological modelling of all graphemes can constitute the Latin script and spatial relationships that may exist between these graphemes to build letters of vocabulary, our studies focused primarily on the Latin printed capital-letter alphabet to test the feasibility of our approach, but it can be generalized to other forms of writing in adopting new forms of graphemes and creating new spatial relations, specific of features vocabularies may exist between concepts.

The reliability of our approach depends on a crucial step is to segment the text document to a set of graphemes, so a good segmentation is already an important step for good recognition, because it is always problematic segmentation and that is why we preferred to manual segmentation by an expert guided to maximize the percentage of expected results. After segmentation and feature extraction, we proposed a normalization step of morphological primitives that based on a classification (each class represents a type of graphemes), this classification will allow us to unify and to appoint all primitives similar under the name of single grapheme, this last going to facilitate us the instantiation of ontology concepts (a concept represents a class of primitives).

\section{ONTOLOGY}

The term ontology originates from philosophy. In that context, it is used as the name of a subfield of philosophy, namely, the study of the nature of existence (the literal translation of the Greek

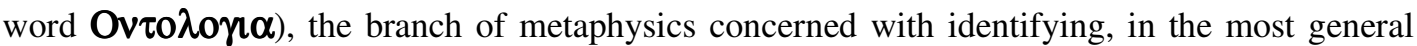
terms, the kinds of things that actually exist, and how to describe them. For example, the observation that the world is made up of specific objects that can be grouped into abstract classes based on shared properties is a typical ontological commitment [2].

However, in more recent years, ontology has become one of the many words hijacked by computer science and given a specific technical meaning that is rather different from the original one. Instead of "ontology" we now speak of "an ontology". For our purposes, we will uses T.R.Gruber's [3] definition, later refined by R.Studer, An ontology is an explicit and formal specification of a conceptualization.

In general, an ontology describes formally a domain of discourse. Typically, an ontology consists of a finite list of terms and the relationships between these terms. The terms denote important 
Signal \& Image Processing : An International Journal (SIPIJ) Vol.4, No.3, June 2013

concepts (classes et objects)of the domain. For example, in a university setting, staff members, students courses, lecture theaters, and disciplines are some important concepts.

The relationships typically include hierarchies of classes. A hierarchy specifies a class $\mathrm{C}$ to be a subclass of another class $\mathrm{C}$ if every object in $\mathrm{C}$ is also included in $\mathrm{C}$.

Apart from subclass relationships, ontologies may include information such as:

- Properties

- Value restrictions

- Disjointness statements

- Specification of logical relationships between objects

In the context of the Web, ontologies provide a shared understanding of a do main. Such a shared understanding is necessary to overcome differences in terminology. One application's zip code may be the same as another application's area code. Another problem is that two applications may use the same term with different meanings.

Ontologies are useful for the organization and navigation of Web sites. Many Web sites today expose on the left-hand side of the page the top levels of a concept hierarchy of terms. The user may click on one of them to expand the subcategories.

Also, ontologies are useful for improving the accuracy of Web searches. The search engines can look for pages that refer to a precise concept in an ontology instead of collecting all pages in which certain, generally ambiguous, keywords occur. In this way, differences in terminology between Web pages and the queries can be overcome.

In addition, Web searches can exploit generalization/specialization information. If a query fails to find any relevant documents, the search engine may suggest to the user a more general query. It is even conceivable for the engine to run such queries proactively to reduce the reaction time in case the User adopts a suggestion. Or if too many answers are retrieved, the search engine may suggest to the user some specializations.

In Artificial Intelligence (AI) there is a long tradition of developing and using ontology languages. It is a foundation Semantic Web research can build upon. At present, the most important ontology languages for the Web are the following:

- XML provides a surface syntax for structured documents but imposes no semantic constraints on the meaning of these documents.

- XML Schema is a language for restricting the structure of XML documents.

- RDF is a data model for objects ("resources") and relations between them; it provides a simple semantics for this data model; and these data models can be represented in XML syntax.

- RDF Schema is a vocabulary description language for describing properties and classes of RDF resources, with a semantics for generalization hierarchies of such properties and classes.

- OWL is a richer vocabulary description language for describing properties and classes, such as relations between classes (e.g., disjointness), cardinality (e.g. "exactly one"), equality, richer typing of properties, characteristics of properties (e.g., symmetry), and enumerated classes [2]. 
Signal \& Image Processing : An International Journal (SIPIJ) Vol.4, No.3, June 2013

\section{SENSORY AND SEMANTIC GAP}

Visual similarity does not necessarily a semantic similarity, Example: two regions in an image of the same color does not mean they represent the same region or the same object. This difference between the conceptual level between machine, which knows only the pixelique data (bridge, line, curve, etc..) And the user who can interpret them (house, bridge, etc.) is called semantic gap. It is the recognition system to bridge this gap by proposing a high-level interpretation of low-level data.

The sensory gap is defined as "the gap between the objects in the real world and the information contained in a description (computer) derived from recording the scene." It is the projection of a reality, 3D and often continues in a 2D discrete computer representation. This gap is to be accepted by researchers working on 2D images, or repelled by researchers working on stereoscopic or 3D images (see figure 1).

The semantic gap is the most difficult to treat. For several years, researchers have revolved around this gap without actually naming it, what is done today. The semantic gap is defined as "the lack of concordance between the information that can be extracted from visual data and the interpretation of these data for a user in a given situation." This gap is more or less the same problem as linking lowlevel treatments and highlevel treatments, except that now it is clearly seen as a problem of information management and not only as a control problem[4].

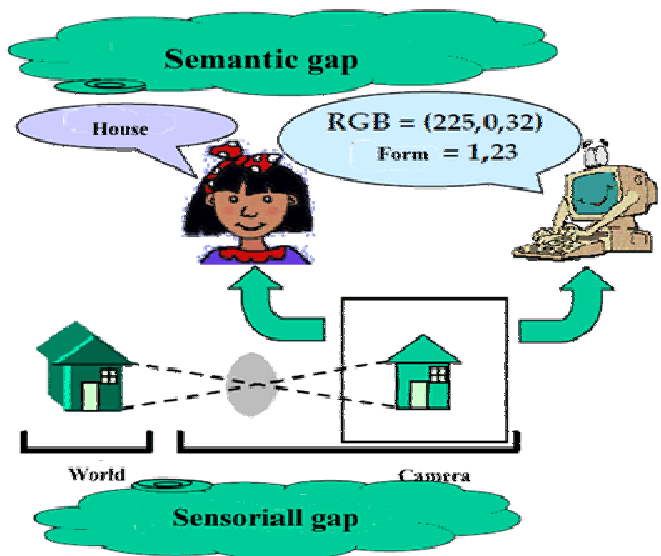

Figure 1. The sensorial gap between the observed world and the acquired image. The semantic gap between computer analysis and human interpretation of the image [5].

In recent years, the gap between semantic concepts and low-level numerical features retained much attention from the scientific community, and remains one of the major challenges in the field of computer vision. To reduce this semantic gap, we have adopted the proposed approach in [6] [7] described by a character recognition system based on ontology and a segmentation of the document processed into graphemes. The types of graphemes that we have adopted are classed in four classes according the classification of philipe Coueignoux [8] (see figure 5).

The ontology plays a role of a bridge between the information represented in the image and knowledge of the expert. Figure 2 illustrates how the information of the image might take a form based on ontologies (left), as well as the knowledge of the expert (right). 
Signal \& Image Processing : An International Journal (SIPIJ) Vol.4, No.3, June 2013

\begin{tabular}{|c|c|c|c|}
\hline $\begin{array}{c}\text { Image information in } \\
\text { pixel matrix }\end{array}$ & $\begin{array}{c}\text { Image information in } \\
\text { ontology }\end{array}$ & $\begin{array}{c}\text { Expert knowledge as } \\
\text { ontological form }\end{array}$ \\
\hline IMAGE & KNOWLEDGE \\
\hline
\end{tabular}

Figure 2. Ontological bridge between image and knowledge

\section{MODELING OF SPATIAL RELATIONS}

Various approaches underlying the modelling of spatial relations, which is a heterogeneous and interdisciplinary field. To model the reality, it is not sufficient to define geographic entities. It should also define spatial relationships between these entities. These spatial relationships are important, especially for knowledge representation, spatial reasoning, and of course, the spatial integrity constraints. They can be classified as follows [9] :

- Relationships based on a spatial order linked to a definition of origin or axis or (eg, direction), - The topological relations that describe neighbourhoods and set-properties (eg, disjoint, adjacent intersects)

- Metric relationships related to distance.

Figure 3, shows the tree of taxonomy of these relationships and table 3 illustrates the detail of RDF/OWL code for each class.

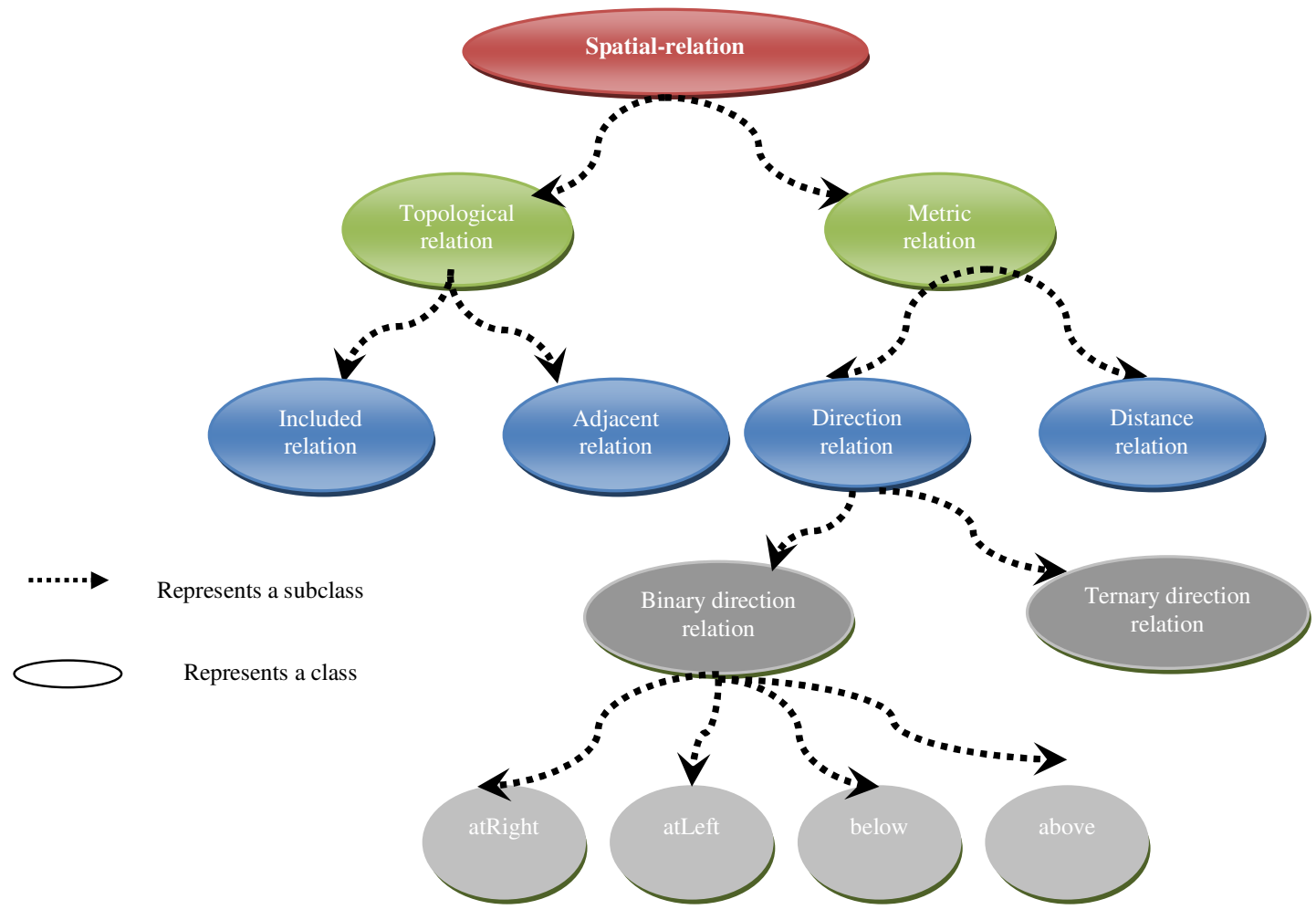

Figure 3. relationships taxonomy 
Signal \& Image Processing : An International Journal (SIPIJ) Vol.4, No.3, June 2013

A spatial relationship allows describing the relative positions between two symbols. For example, given the expression $\boldsymbol{a} \boldsymbol{b}$, the symbols $\boldsymbol{a}$ and $\boldsymbol{b}$ are connected by the relation left/right. While they are connected by the relation (top-right/bottom-left) in the expression $\boldsymbol{a}^{b}$. In most systems proposed a relationship bears more structural sense a semantic meaning (logical relation). For example, the structural relationship in $\boldsymbol{a}^{b}$ means mathematically that $\boldsymbol{a}$ to the power $\boldsymbol{b}[\mathbf{1 0}]$.

Figure 4 shows an extract of the spatial taxonomy implemented under the Protégé2000 editor. This tree shows the spatial relationships of the Latin alphabet, but we can enrich it and reuse it, for other types of alphabet (example: Arabic, Chinese, etc).

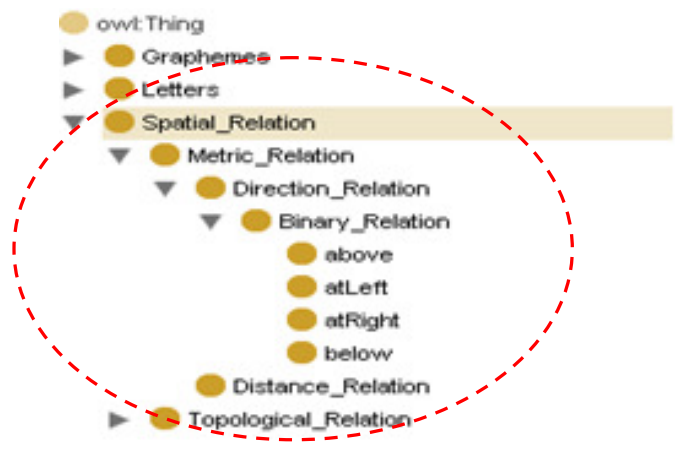

Figure 4. Relationships taxonomy under Protégé2000

The types of relationships that we addressed in our study are the following relations:

- adjacency between two segments or two objects of interest or two semantic objects (distinguishing adjacencies top, bottom, right and left): "a Bar is adjacent to a Trunk and is located to the right of it (the case of letter ' $\mathbf{L}$ '). "

- Neighbourhood between two segments or two objects of interest or two semantic objects without contact between them (distinguishing cases north, south, east, west) "a Point is located north of Trunk" (the case of the letter 'i') (see figure 7).

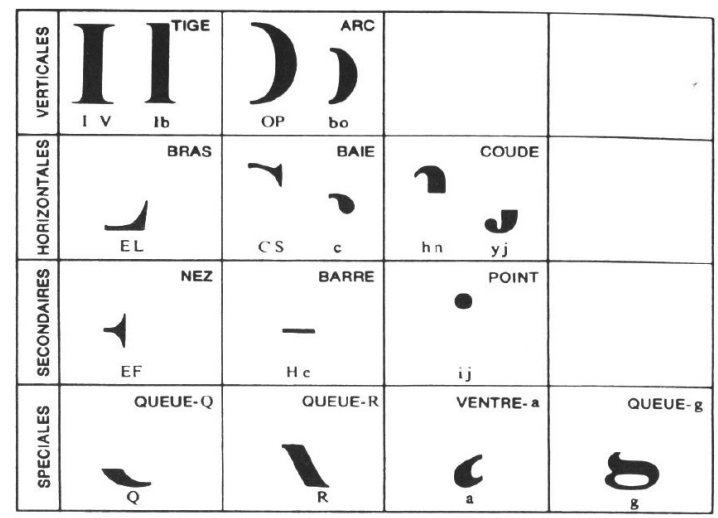

Figure 5. Classification of graphemes by philipe Coueignoux

According to the classification of philipe Coueignoux, we created taxonomy of graphemes, where each of them is represented by a concept bearing his name. Figure 5 shows an excerpt of this 
Signal \& Image Processing : An International Journal (SIPIJ) Vol.4, No.3, June 2013

taxonomy published under the ontologies Editor: Protégé2000 (The Protégé Ontology Editor and Knowledge Acquisition System: http://protege.stanford.edu/)

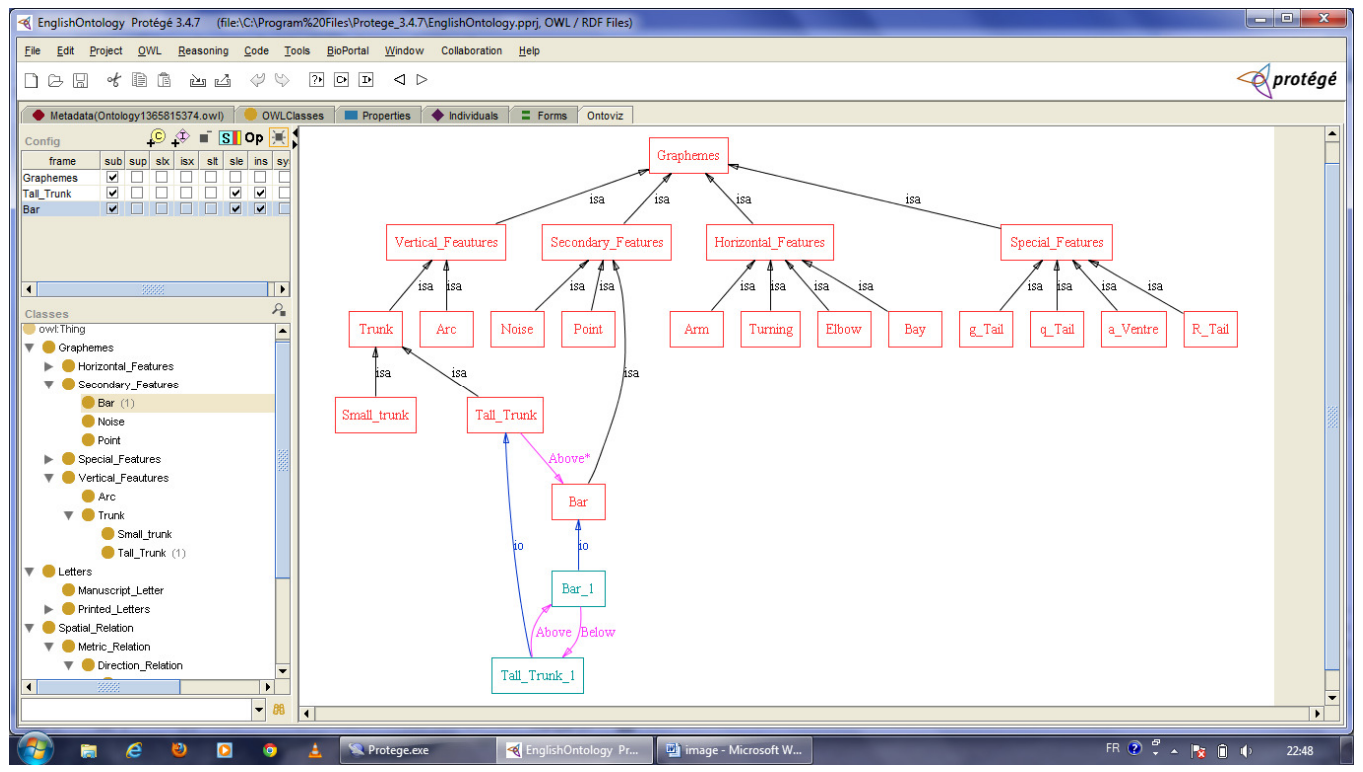

Figure 6. Taxonomy of graphemes

Figure 7, shows an example of segmented character in two graphemes such as "Small_Trunk" and "Point" who they are connected by a spatial relation : "Above", you can also note that this last can have an inverse relationships which is "Below", it is possible to implement this type of relations with Protégé2000.

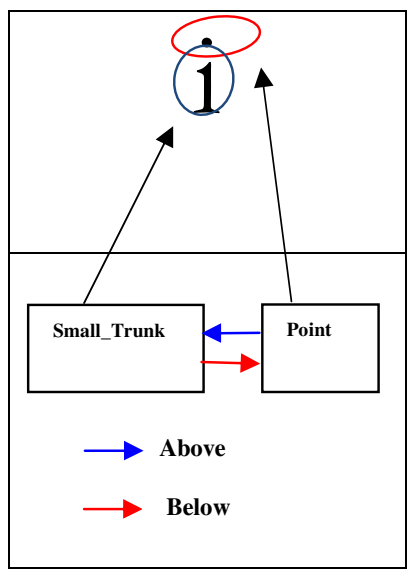

Figure 7. graphemes and spatial relations composing the character "i"

and the RDF/OWL code of this relationship is mentioned here by this code:

<owl:ObjectProperty rdf:ID="Below">

<owl:inverseOf rdf:resource="\#Above"/>

$</$ owl:ObjectProperty> 
Signal \& Image Processing : An International Journal (SIPIJ) Vol.4, No.3, June 2013

Figure 8, shows via the plugin Ontoviz of the protégé2000 editor, an example of an instantiation of the concepts "Small_Trunk" and "Point" and the relationships "above, Below" for building of character "i".

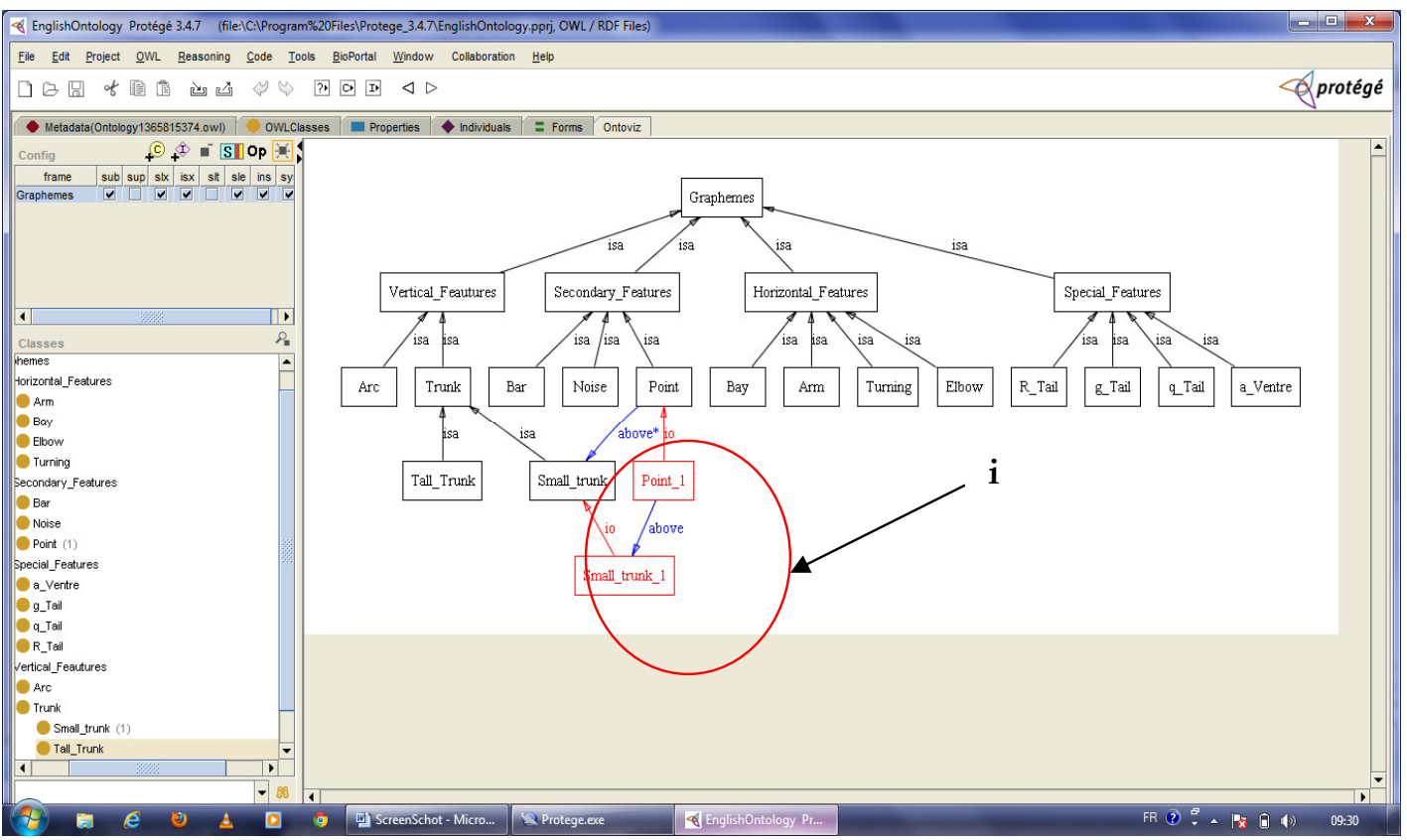

Figure 8. An example of ontology instantiation (Case of character : i)

\section{IMPLEMENTATION AND RESULT}

Protégé has an open architecture that allows programmers to integrate plug-ins, which can appear as separate tabs, specific user interface components (widgets), or perform any other task on the current model. The complete source code for most of the examples can be found in the edu.stanford.smi.protegex.owlx.examples package in the Protégé-OWL source code [11]. if you use Eclipse or Netbeans as a Java IDE, it must select the Protégé installation folder as your project home, then add all the JAR files from the installation to your project class path.

Protégé-OWL API is centered around a collection of Java interfaces from the model package that provide access to the OWL model and its elements (classes, properties, and individuals). The most important interface is OWLModel which provides access to the top-level container of the resources in the ontology so you can use OWLModel to create, query, and delete resources of various types and then use the objects returned by the OWLModel to do specific operations [12]. Table 1, shows the example for creating Spatial-relation class with java code.

Table 1. Java code for creating Spatial-relation class:

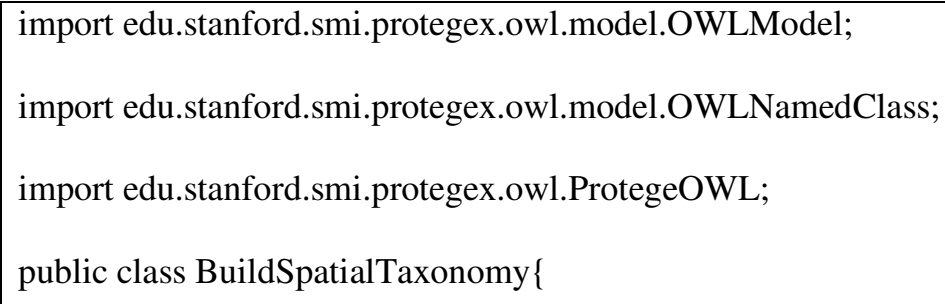


Signal \& Image Processing : An International Journal (SIPIJ) Vol.4, No.3, June 2013

public static void main(String[] args) \{

OWLModel owlModel= ProtegeOWL.createJenaOWLModel();

owlModel.getNamespaceManager().setDefaultNamespace("http://SpatialTaxonomy.com\#");

OWLNamedClass spatialClass $=$ owlModel.createOWLNamedClass

("Spatial-relation"); \}

System.out.println("Class URI: " + spatialClass.getURI());

\}

In the table 2, we have outlined the most important primitives that allow read and browse the OWL ontologies. The package "com.hp.hpl.jena.ontology" provides a set of classes and methods to manipulate and access to this type of ontology.

Table 2. Primitives of manipulating the OWL ontologies

\begin{tabular}{|c|c|}
\hline Role & Statement \\
\hline Creating an ontology model $\mathbf{~ m . ~}$ & $\begin{array}{l}\text { OntModel m=ModelFactory.createOntologyModel } \\
\text { (OntModelSpec.OWL_MEM,null); }\end{array}$ \\
\hline $\begin{array}{l}\text { Load and creation a copy of } \\
\text { ontology }\end{array}$ & m.getDocument Manager. addAltEntry() \\
\hline Reading of ontology & m.read ( ); \\
\hline $\begin{array}{l}\text { Browse the ontology classes. } \\
\text { Give all classes of ontology }\end{array}$ & $\begin{array}{l}\text { for (Iterator } \mathrm{i}=\mathrm{m} . \text { listClasses }() \text {; i.hasNext }()) \text {; } \\
\{\text { OntoClass cls }=(\text { OntClass }) \text { i.next }() ;\end{array}$ \\
\hline Local name of class & $\begin{array}{l}\text { getLocalName () } \\
\text { System.out.println(cls.getLocalName); }\end{array}$ \\
\hline Browse the super classes of cls & $\begin{array}{l}\text { for (Iterator i =cls. listSuper.Classes( ); i .haslNext()) } \\
\{\text { Sysem.out.print }(((\text { OntClass)i.next()).getLocalName() } \\
+“ " ”) ;\}\end{array}$ \\
\hline Browse the subclasses of cls & $\begin{array}{l}\text { for (Iterator i =cls. listSub. Classes( }) \text {; i .haslNext()) } \\
\{\text { Sysem.out.print }(((\text { OntClass)i.next()).getLocalName }() \\
+ \text { “"” }) ;\}\end{array}$ \\
\hline
\end{tabular}

Table 3. Implementation code RDF/OWL of relationships taxonomy

\begin{tabular}{|c|l|}
\hline $\begin{array}{c}\text { Concepts of relationship } \\
\text { Taxonomy }\end{array}$ & Code Rdf/OWL \\
\hline Spatial-relation & $\begin{array}{l}<\text { owl:Class rdf:ID="Spatial_Relation"/> } \\
</ \text { owl:Class }>\end{array}$ \\
\hline & $<$ owl:Class rdf:ID="Metric_Relation" $>$ \\
\hline
\end{tabular}


Signal \& Image Processing : An International Journal (SIPIJ) Vol.4, No.3, June 2013

\begin{tabular}{|c|c|}
\hline Metric relation & $\begin{array}{l}\text { <rdfs:subClassOf } \\
\text { rdf:resource="\#Spatial_Relation"/> } \\
</ \text { owl:Class }>\end{array}$ \\
\hline Direction relation & $\begin{array}{l}\text { <owl:Class rdf:ID="Direction_Relation"> } \\
\quad \text { <rdfs:subClassOf } \\
\text { rdf:resource="\#Metric_Relation"/> } \\
\quad \text { </owl:Class }>\end{array}$ \\
\hline Distance relation & $\begin{array}{l}\text { <owl:Class rdf:ID="Distance_Relation"> } \\
\quad \text { <rdfs:subClassOf } \\
\text { rdf:resource="\#Metric_Relation"/> } \\
\quad \text { </owl:Class }>\end{array}$ \\
\hline Binary relation & $\begin{array}{l}\text { <owl:Class rdf:ID="Binary_Relation"> } \\
\quad \text { <rdfs:subClassOf } \\
\text { rdf:resource="\#Direction_Relation"/> } \\
\quad \text { </owl:Class }>\end{array}$ \\
\hline atRight & $\begin{array}{l}\text { <owl:Class rdf:ID="atRight"> } \\
\quad \text { <rdfs:subClassOf } \\
\text { rdf:resource="\#Binary_Relation"/> } \\
\quad \text { </owl:Class }>\end{array}$ \\
\hline atLeft & $\begin{array}{l}\text { <owl:Class rdf:ID="atLeft"> } \\
\quad \text { <rdfs:subClassOf } \\
\text { rdf:resource="\#Binary_Relation"/> } \\
\quad \text { </owl:Class }>\end{array}$ \\
\hline below & $\begin{array}{l}\text { <owl:Class rdf:ID="below"> } \\
\quad \text { <rdfs:subClassOf } \\
\text { rdf:resource="\#Binary_Relation"/> } \\
\quad \text { </owl:Class }>\end{array}$ \\
\hline above & $\begin{array}{l}\text { <owl:Class rdf:ID="above"> } \\
\quad \text { <rdfs:subClassOf } \\
\text { rdf:resource="\#Binary_Relation"/> } \\
\quad \text { </owl:Class > }\end{array}$ \\
\hline Adjacent relation & $\begin{array}{l}\text { <owl:Class rdf:ID="Adjacent_Relation"> } \\
\quad \text { <rdfs:subClassOf } \\
\text { rdf:resource="\#Topological_Relation"/> } \\
\text { </owl:Class }>\end{array}$ \\
\hline Included relation & $\begin{array}{l}\text { <owl:Class rdf:ID="Inclued_Relation"> } \\
\quad<\text { rdfs:subClassOf } \\
\text { rdf:resource="\#Topological_Relation"/> } \\
\quad \text { </owl:Class }>\end{array}$ \\
\hline Topological Relation & $\begin{array}{l}\text { <owl:Class rdf:ID="Topological_Relation"> } \\
\quad<\text { rdfs:subClassOf } \\
\text { rdf:resource="\#Spatial_Relation"/> } \\
\quad<\text { /owl:Class }>\end{array}$ \\
\hline
\end{tabular}

\section{Conclusions}

In this paper, we presented a new paradigm for character recognition problem based on the domain ontology that is characterised by description of spatial relations between graphemes. These last are represented by ontological concepts and the relations linking them are of spatial type describing their spatiality in the document processed. In this article we haven't published all type of relations but only the most important. 
Signal \& Image Processing : An International Journal (SIPIJ) Vol.4, No.3, June 2013

The development of Ontologies in this area can be used to provide image analysis automation support and efficient use of modern methods and techniques for image analysis and pattern recognition.

In the future work, we plan to expand and enrich the taxonomy of relationships with other types of relationships and add SWRL inference rules to strengthen the reasoning on our ontology. We plan to use the matching operations with external resources such as WordNet by using dedicated similarity measures in the step of post-treatment.

\section{REFERENCES}

[1] Ali Borji • Mandana Hamidi - Fariborz Mahmoudi, (2008) "Robust Handwritten Character Recognition with Features Inspired by Visual Ventral Stream”, Neural Processing Letters 28(2): 97 111.

[2] Grigoris Antoniou and Frank van Harmelen, (2004) “A Semantic Web Primer", TLFeBOOK, The MIT Press.

[3] T.R. Gruber, (1993) "A Translation approach to Portable Ontology Specification Knowledge Acquisition”, International Journal of Knowledge Acquisition for Knowledge-based systems, Vol. 5, Num. 2.

[4] A.W.M. Smeulders, M. Worring, S.Santini, A.Gupta and R.Jain, (2000) "Content-Based Image Retrieval at the End of the Early Years". IEEE Trans. on Patt. Anal. and Machine Intell., Vol. 22, Num. 12, pp. 1349-1380.

[5] Alain Boucher and Thi-Lan Le, (2005) "Comment extraire la sémantique d'une image ?", SETIT 2005, 3rd International Conference: Sciences of Electronic, Technologies of Information and Telecommunications.

[6] Hacene Belhadef and Aicha Eutamene, (2012) "Ontology and character recognition : New axis of research, International journal of academic research Vol. 4. Num. 2.

[7] Aicha Eutamene, Hacene Belhadef and M. K. Kholladi, (2011) "New Process Ontology-Based Character Recognition", LNCS Series of Communications in Computer and Information Science, Vol. 240.

[8] Ph. Coueignoux, (1983) “Approche structurelle de la lettre In: Langue française”, N59, pp. 45-67.

[9] Marc Souris, "Les principes et algorithmes des systèmes d'information géographique L'exemple du système SavGIS", Chapter 7 : "Contraintes d"'intégrité spatiale et saisie graphique",

[10] Ahmad Montaser AWAL, (2010) "Reconnaissance de structures bidimensionnelles : Application aux expressions mathématiques manuscrites en-ligne", Thèse de Doctorat de l'Ecole polytechnique de l'Université de Nantes.

[11] Protege-OWL API Programmer's Guide, URL: http://protegewiki.stanford.edu/wiki/ProtegeOWL_API_Programmers_Guide

[12] Working with OWL Models, URL: http://protegewiki.stanford.edu/wiki/ProtegeOWL_API_Basics 
Signal \& Image Processing : An International Journal (SIPIJ) Vol.4, No.3, June 2013

\section{Authors}

Aicha Eutamene is Phd student at NTIC Faculty, University of Constantine 2-Algeria.

Mohamed Khireddine Kholladi, Professor at NTIC Faculty, University of Constantine 2-Algeria, President of MISC Laboratory

Hacene Belhadef, Associate professor at NTIC Faculty, University of Constantine 2-Algeria, Member of MISC Laboratory (corresponding author email: hacene_belhadef@yahoo.fr) 